Den aktuellen Stand möglicher

\section{Bemerkungen zur Arbeit von G. Schreiber und A. Wilmer Varikozele und testikuläre Funktionsstörungen?}

Reproduktionsmedizin (1998) 14: 251-256

E. Nieschlag

Zentrum für Frauenheilkunde, Institut für Reproduktionsmedizin der Westfälischen Wilhelms-Universität Münster

Schreiber und Wilmer übergehen in ihrem Artikel „Varikozele und testikuläre Funktionsstörungen?" die aktuelle Diskussion über Therapiekonzepte bei Varikozele. Die Autoren empfehlen nach wie vor die interventionelle Therapie der Varikozele (chirurgische Ligatur oder angiographische Okklusion) und nennen als Indikation für diese Verfahren normabweichende Spermiogramme und ein verkleinertes ipsilaterales Hodenvolumen. Hierbei beziehen sie sich auf eine Empfehlung der WHO aus dem Jahr 1992, verschweigen aber, daß inzwischen neue Erkenntnisse vorliegen.

So kommt eine kürzlich veröffentlichte Metaanalyse der bisher vorliegenden und im Sinne der ,evidencebased medicine" auswertbaren, d.h.

Prof. Dr. E. Nieschlag

Reproduktionsmedizin, Domagkstraße 11, D-48129 Münster kontrollierten und randomisierten Studien zu dem Schluß, daß die interventionelle Therapie der Varikozele nicht zu einer Erhöhung der Schwangerschaftsraten führt (Evers 1998). Bei der Betreuung des infertilen Paares ist sicher die Erzielung einer Schwangerschaft wichtigster Endpunkt der Behandlung und nicht eine mögliche Verbesserung der Spermienzahl, ein Verschwinden des Rückflusses oder eine Zunahme des Hodenvolumens. In einer eigenen kontrollierten randomisierten Studie zur Behandlung der Varikozele, die mit 125 Paaren die bisher größte unizentrische Studie zu dieser Problematik darstellt, kommen wir zu dem Schluß, daß die konservative Betreuung des infertilen Paares zu einer ebenso hohen Schwangerschaftsrate führt wie eine interventionelle $\mathrm{Be}$ handlung der Varikozele (Nieschlag et al. 1998). Dabei vertreten wir nicht einen therapeutischen Nihilismus. Im Gegenteil, unsere Studie zeigt, daß die intensive Betreuung des Patienten und des Paares eine interventionelle Therapie ersetzen kann.
Therapieempfehlungen faßt das britische Royal College of Obstetricians \& Gynaecologists (RCOG) in seinen publizierten Richtlinien zur Therapie der Infertilität wie folgt zusammen: Recommendation 6: „There is insufficient evidence to recommend occlusion of the left internal spermatic vein in subfertile or oligozoospermic men with varicocele" (Templeton et al. 1998).

Selbst renommierte deutsche Andrologen, die die operative Seite vertreten, stellen inzwischen den Wert der interventionellen Therapie in Frage (Weidner 1998).

Ich meine, daß diese aktuelle Diskussion den Lesern der „Reproduktionsmedizin“ nicht vorenthalten werden sollte.

\section{Literatur}

Evers JLH (1998) Varicocele. In: Templeton A, Cooke I, O'Brien PMS (eds) Evidencebased fertility treatment. RCOG Press, London

Nieschlag E, Hertle L, Fischedick A, Abshagen K, Behre HM (1998) Update on treatment of varicocele: counselling as effective as occlusion of the vena spermatica. Hum Reprod 13: 2147-2150

Schreiber G, Wilmer A (1998) Varikozele und testikuläre Funktionsstörungen? Reproduktionsmedizin 14: 251-256

Templeton A, Cooke I, O'Brien PMS (eds) (1998) Evidence-based fertility treatment. RCOG Press, London, p399

Weidner W (1998) Therapie der Varikozele: Versuch einer Standortbestimmung. Urologe 37: 277-281 